\title{
Estudio comparativo de la eficacia de dos técnicas de HDF on line
}

\author{
Carmen Terry Osset* \\ Mateo Párraga Díaz* \\ Eulalia Cárceles Legáz* \\ Esperanza Melero Rubio* \\ Francisco Belchí Rosique** \\ Felicidad Astorga Pérez** \\ Josefina Chica Arellano**
}

* Hospital Universitario Virgen Arrixaca (Murcia)

** Hospital St ${ }^{\underline{a}}$ Maria del Rossell (Cartagena)

\section{RESUMEN}

Existen dos técnicas Hemodiafiltración (HDF) on line: predilucional y posdilucional, siendo esta última la más utilizada. Nuestro estudio compara la eficacia de dos técnicas de HDF, posdilucional y mixta, analizando la frecuencia de casos en los que existió un aumento de la presión transmembrana, hecho que impidió continuar con el tratamiento.

El estudio constó de dos fases, en la primera fase, (HDF on line posdilucional), reducíamos la tasa de infusión (Qi) cada vez que la presión transmembrana (PTM) era $\geq 350 \mathrm{mmHg}$. y pasabamos a hemodiálisis convencional si Qi era $30 \mathrm{ml} / \mathrm{min}$. En la segunda fase, (HDF on line mixta), cambiábamos a predilucional durante la última hora y cada vez que la PTM era 350 $\mathrm{mmHg}$.

En la fase 1 apareció hemoconcentración (HC) en un $38.83 \%$ de las sesiones y en la fase 2 en un $18.06 \%$. En la última hora de tratamiento, la HC apareció en 119 casos en la fase 1 y en 15 casos en la fase 2 . Se produjo la coagulación del sistema en 24 ocasiones

\section{Correspondencia:}

Carmen Terry Osset

Servicio Nefrología, Sección Diálisis

Hospital Universitario Virgen de la Arrixaca

Ctra. Madrid-Cartagena, $s / n$

30120 El Palmar (Murcia) durante la fase 1 y en 2 ocasiones durante la fase 2. Se produjeron hipotensiones en 80 ocasiones en la fase 1 y en 69 en la fase 2.

Ante el aumento de PTM durante la HDF on line posdilucional la técnica mixta resultó más eficaz que continuar en posdilucional con disminución del Qi. Con el método mixto fue mayor la eliminación de $\beta_{2}$ microglobulina y no disminuyó la eficacia de la técnica.

\section{PALABRAS CLAVE: HEMODIAFILTRACIÓN ON LINE PRESIÓN TRANSMEMBRANA EFICACIA HEMOCONCENTRACIÓN}

\section{COMPARATIVE STUDY OF THE EFFICACY OF TWO ONLINE HDF TECHNIQUES}

\begin{abstract}
There are two online haemodiafiltration (HDF) techniques: pre-dilutional and post-dilutional, the latter being the most frequently used. Our study compares the efficacy of two HDF techniques, post-dilutional and mixed, analysing the frequency of cases in which there was an increase in trans-membrane pressure, preventing continuation of the treatment.

The study consisted of two phases, in the first phase, (post-dilutional online HDF), we reduced the infusion rate $(\mathrm{Q} i)$ every time the trans-membrane pressure
\end{abstract}


(TMP) was $\geq 350 \mathrm{mmHg}$ and we moved on to conventional haemodialysis if Qi was $30 \mathrm{ml} / \mathrm{min}$. In the second phase, (mixed online HDF), we changed to pre-dilutional during the last hour and every time the TMP was $\geq 350 \mathrm{mmHg}$.

In phase 1 haemoconcentration $(\mathrm{HC})$ appeared in $38.83 \%$ of sessions and in phase 2 in $18.06 \%$. During the last hour of treatment, $\mathrm{HC}$ appeared in 119 cases in phase 1 and in 15 cases in phase 2. Coagulation of the system occurred on 24 occasions during phase 1 and on 2 occasions during phase 2. Hypotension occurred on 80 occasions in phase 1 and on 69 in phase 2.

In light of the increase in TMP during post-dilutional HDF, the mixed technique proved more efficient than continuing in post-dilutional with a reduction of $\mathrm{Qi}$. With the mixed method the elimination of de $\beta_{2}$ microglobulin was greater and the efficacy of the technique was not reduced.

KEY WORDS:

ONLINE HAEMODIAFILTRATION
TRANS-MEMBRANE PRESSURE
EFFICACY
HAEMOCONCENTRATION

\section{INTRODUCCIÓN}

Las terapias sustitutivas renales deberían imitar la función de un riñón normal. Sin embargo, es difícil de conseguir por la naturaleza discontinua del tratamiento y por las características de aclaramiento del riñón artificial ${ }^{(1)}$.

Entre los tratamientos dialíticos actuales, la HDF on li$n e$, es una técnica que ofrece algunas ventajas con respecto a la hemodiálisis convencional, como son: una mayor depuración de pequeños y grandes solutos, al combinar en la transferencia los mecanismos de difusión y convección ${ }^{(2,3)}$, ya que se utilizan tasa altas de ultrafiltración (6-12 litros/hora), que se compensan con la infusión on line de líquido de reposición; y con mayor biocompatibilidad, ya que el líquido de infusión es ultrapuro, con todas las ventajas que ello conlleva.

Hay dos maneras de llevar a cabo la técnica de HDF on line: predilucional (PRE) y posdilucional (POS). En el método PRE, la sangre sale del paciente y se mezcla con el líquido de reposición antes del dializador, mientras que en el POS, la sangre que sale del paciente entra en el dializador eliminando gran parte del líquido plasmático y, en el retorno venoso, se perfunde el líquido de reposición en la misma cantidad que se ha eliminado menos el volumen de peso que se quiere extraer al paciente. Experiencias in vitro e in vivo demuestran claramente una mayor eficacia depurativa para la $\mathrm{POS}^{(4)}$. La PRE tiene menor eficacia en la depuración de pequeños solutos como consecuencia del menor transporte difusivo y tiene mejor aclaramiento de $\beta_{2}$ microglobulina por el mayor transporte convectivo ${ }^{(5)}$.

Sin embargo, nos encontramos con unos inconvenientes propios de la HDF on line POS como el aumento de la PTM y, por tanto, la posibilidad de coagulación del sistema $^{(1,6,7)}$. En algunas unidades han solucionado este problema con el uso de un monitor al que se le ha incorporado un sistema automático, el cual, cambia de HDF on line posdilucional a predilucional cuando los rangos de seguridad lo requieren, manteniendo la PTM por debajo de los $300 \mathrm{mmHg}$, alcanzándose de esta forma la mayor eficacia ${ }^{(7)}$. De igual manera, se está estudiando dializadores que permiten HDF mixtas con el mayor rendimiento posible $e^{(8,9)}$.

En nuestras unidades, como en la mayoría donde se utiliza la técnica HDF on line, no se dispone de ningún dispositivo similar al descrito anteriormente y, por lo tanto, necesitamos de una mayor atención de enfermería durante el tiempo que dura el tratamiento, para evitar que el aumento de la PTM ponga en peligro la seguridad de la técnica. Por ello, y partiendo de la premisa de que la HDF on line POST es el método más cercano al ideal( ${ }^{(4)}$, nuestro estudio se centra en analizar cuál sería el método de elección en el caso de que la PTM se elevara por encima de los rangos de seguridad establecidos ( $\geq 350 \mathrm{mmHg}$ ) y no se pudiera mantener la HDF on line POST durante toda la sesión.

- Método 1: Disminuir la tasa de infusión (Qi) manteniendo durante toda la sesión el método Posdilucional

- Método 2: Pasar a PRE haciendo una HDF mixta.

\section{OBJETIVOS}

El objetivo general de este estudio se centra en comparar la eficacia de las técnicas de HDF on line, anterior- 
mente enunciadas: Posdilucional y Mixta. Para ello nos planteamos los siguientes objetivos específicos:

1. Analizar en ambas fases la frecuencia de casos en los que el aumento de PTM $\geq 350 \mathrm{mmHg}$, nos impide realizar la técnica pautada.

2. Identificar qué parámetros, analíticos y de procedimiento, pueden verse alterados, según el tipo de tratamiento utilizado.

\section{MATERIAL Y MÉTODO}

Se realizó un estudio descriptivo prospectivo cuya duración fue de 16 semanas dividido en dos fases, ocho semanas en tratamiento de HDF on line con una técnica postdilución y ocho semanas con técnica mixta. Se llevó a cabo en dos centros hospitalarios de la región incluyendo a todos los pacientes que en el momento del inicio estaban recibiendo tratamiento de HDF on line, en dos tipos de monitor diferentes, Fresenius $4008 \mathrm{H}^{\circledR} \mathrm{y}$ Fresenius $4008 S^{\circledast}$. Se recogieron datos referentes tanto a los parámetros de la técnica como a los parámetros analíticos.

Fase 1: Con técnica POS, al alcanzar la PTM un valor de $350 \mathrm{mmHg}$, se disminuía la tasa de intercambio del líquido de sustitución en $30 \mathrm{ml} / \mathrm{min}$. A continuación, cada vez que alcanzábamos dicha cifra de PTM se disminuía la tasa de $20 \mathrm{ml} / \mathrm{min}$ en $20 \mathrm{ml} / \mathrm{min}$. Cuando la tasa de intercambio era inferior a $30 \mathrm{ml} / \mathrm{min}$ se pasaba a diálisis convencional.

Fase 2: La que denominamos mixta, comenzamos la sesión en técnica POS y se pasaba a PRE en aquellas ocasiones en las que aumentaba la PTM hasta el límite máximo marcado de $350 \mathrm{mmHg}$ manteniendo la misma tasa de intercambio. En todas las ocasiones, y si antes no se había cambiado, al inicio de la última hora del tratamiento de HDF on line realizamos la misma maniobra de cambio.

Por tanto, llamaremos hemoconcentración (HC), a la elevación de la PTM hasta el valor de $\geq 350 \mathrm{mmHg}$ y consideramos hipotensión cuando la situación del paciente requirió alguna medida correctora.

También se realizaron controles analíticos en la primera, cuarta, octava, duodécima y decimosexta semana. Se extrajeron muestras de sangre prediálisis para determinar: proteínas totales, albúmina, triglicéridos, coleste- rol, hematocrito, hemoglobina, calcio, fósforo, control de coagulación, Dimero $\mathrm{D}, \beta_{2}$ microglobulina y $\mathrm{Kt} / \mathrm{v}$ URR y, tras 15 minutos iniciada la sesión, se tomó nueva muestra para determinar recirculación del injerto (test de recirculación).

El análisis estadístico se realiza a través del programa SPSS 13.0 para Windows. Para las variables cuantitativas utilizamos medias y desviación típica, para las variables cualitativas fracciones y porcentajes. Aplicamos la "t" de Student para el contraste de medias en muestras relacionadas con variables cuantitativas independientes, Chi cuadrado para variables cualitativas dependientes e independientes.

\section{Análisis de la muestra}

Se estudió un total de 21 pacientes, acabando 19 el estudio (uno no finalizó por problemas en el acceso vascular y otro por pérdida del seguimiento). La edad media era de $58.21 \pm 11.53$ años, 12 hombres (63.2\%) y 7 mujeres (36.8\%). El tiempo de permanencia en hemodiálisis era de $89 \pm 96.07$ meses, el tiempo en tratamiento on line $13.11 \pm 10.05$ meses. La causa de la insuficiencia renal: 5 diabetes (26.3\%), 5 desconocida (26.3\%), 2 HTA (10.5\%), 2 intersticial (10.5\%), 1 glomerular (5.3\%), 1 enf. poliquística (5.3\%) y 3 otras causas (15.8\%).

La duración de las sesiones era de cuatro horas. El tipo de dializador utilizado: en 15 pacientes polisulfona (78.9\%), en un 1 paciente polietersulfona (5.3\%), en 3 pacientes poliariletersulfona (15.8\%). La heparinización del circuito extracorpóreo en 16 casos heparina de bajo peso molecular (84.2\%) y en 3 casos heparina sódica (15.8\%). El flujo sanguíneo medio en la fase 1 era $326.16 \pm 15.8$ y en la fase 2 era $330.17 \pm 9.37$, con un flujo de infusión (Qi)=1/3 flujo sanguíneo-10.

Los pacientes que estaban antiagregados fueron 13 (68.4\%), de ellos 11 con clopidogrel y 2 con AAS, y 6 que no lo estaban (31.6\%). El peso seco medio 66.69 \pm 13.31 $\mathrm{Kg}$.

\section{RESULTADOS}

En la fase 1, sobre 493 sesiones válidas (13 excluidas por averías en el monitor), en 172 se produce $\mathrm{HC}$, lo que representa un 38,83\% del total de las sesiones. En la fase 2, sobre 432 sesiones válidas (24 excluidas por 
avería en el monitor), en 78 se produce $\mathrm{HC}$, lo que representa un 18,06\% de las sesiones. Con diferencia estadísticamente significativa $\mathrm{p}<0,001$ (tabla 1 ).

\section{Tabla 1. Sesiones con HC}

\begin{tabular}{l|l|l|l|} 
& № S. válidas & № $\mathrm{HC}$ & $\% \mathrm{HC}$ \\
\hline Fase 1 & 493 & 172 & $38,83 \%$ \\
\hline Fase 2 & 432 & 78 & $18,06 \%$ \\
\hline P & & $\mathrm{p}<0,001$ & \\
\hline
\end{tabular}

Tabla 1.

Sobre las frecuencias de HC, expresadas por fracciones horarias, observamos que es mayor la $\mathrm{HC}$ en la fase 1 que en la fase 2 sobre todo en la tercera y cuarta hora. Con diferencia estadísticamente significativa $(p<0,001)$ en la última hora del tratamiento (tabla 2).

\section{Tabla 2. Frecuencia HC}

\begin{tabular}{|l|l|l|l|l|} 
& 1h & $2 \mathrm{~h}$ & $3 \mathrm{~h}$ & $4 \mathrm{~h}$ \\
\hline Fase 1 & 14 & 39 & 50 & 119 \\
\hline Fase 2 & 10 & 26 & 29 & 15 \\
\hline P & ns & ns & $=0.020$ & $<0.001$
\end{tabular}

Tabla 2.

Las veces que tuvimos que pasar de $\mathrm{HDF}$ on line a $\mathrm{HD}$ convencional, por problemas de HC: 31 veces en la fase $1,(7 \%)$, frente a las 3 veces en la fase $2(0.23 \%)$. Con diferencia estadísticamente significativa $(p<0.001)$. Se produjo coagulación del sistema: 24 ocasiones en la fase $1(5.42 \%)$, frente a las 2 en la fase $2(2.08 \%)$, con diferencia estadísticamente significativa $(p<0.012)$. En cuanto a la frecuencia de hipotensiones: en la fase 1 fueron 80 (18.06\%) y en la fase 2 fueron 69 (15.97\%), sin diferencia estadística.

Los parámetros del monitor observados no indicaron variación significativa excepto en el número de $\mathrm{HC}$ por sesión que fue mayor en la fase 1 y los litros de intercambio que eran mayores en la fase 2 (tabla 3 ).

En cuanto a los resultados analíticos resaltamos que no hubo variación significativa excepto en la eliminación de $\beta_{2}$ microglobulina que fue mayor en la fase 2 (tabla 4).

La TA media en prediálisis, fue de $92.05 \pm 21.37 \mathrm{mmHg}$ en la fase 1 , frente a $95.45 \pm 9.62 \mathrm{mmHg}$ en la fase 2 , sin significación estadística. El número de hipotensiones por sesión fue de $0.27 \pm 0.42$ en la fase 1 , frente $0.25 \pm 0.52$ en la fase 2 , sin significación estadística.

Tabla 3. Parámetros del monitor

\begin{tabular}{|c|c|c|c|c|c|c|c|}
\hline & $Q B$ ml/min. & QI ml/min. & UFt $m l$. & HC Ses. & PTM mmHg & PVM mmHg & L. Inter. \\
\hline Fase 1 & $326.16 \pm 15.8$ & $95.06 \pm 3.95$ & $2429.56 \pm 587.88$ & $0.57 \pm 0.59$ & 303.7 & 189.4 & $20.62 \pm 2.35$ \\
\hline \multirow[t]{2}{*}{ se 2} & $330.17 \pm 9.37$ & $96.42 \pm 2.70$ & $2331.67 \pm 542.46$ & $0.19 \pm 0.20$ & $226.89 \pm 35.30$ & $183.44 \pm 25.41$ & $22.70 \pm 1.12$ \\
\hline & ns & ns & ns & $p<0.001$ & $\mathrm{p}=0.019$ & ns & $\mathrm{p}<0.001$ \\
\hline
\end{tabular}

Tabla 4. Resultados analíticos

\begin{tabular}{|llllllll|}
\hline & Recircul. & $\mathrm{Kt} / \mathrm{v}$ & $\beta 2 \mathrm{mg} / \mathrm{dl}$ & $\mathrm{Hb}$ gr/dl & Dimero D mg/d & P.T. gr/dl & Htc \% \\
\hline Fase 1 & $4.31 \pm 2.68 \%$ & $1.64 \pm 0.18 \%$ & $27279.87 \pm 6558.89$ & $12.49 \pm 1.59$ & $289.19 \pm 155.03$ & $6.75 \pm 0.43$ & $37.67 \pm 5.16$ \\
\hline Fase 2 & $4.19 \pm 2.52 \%$ & $1.66 \pm 0.21 \%$ & $22122.16 \pm 5409.77$ & $12.91 \pm 1.64$ & $281.17 \pm 104.02$ & $6.72 \pm 0.46$ & $37.58 \pm 5.57$ \\
\hline & ns & ns & $\mathrm{P}=0.003$ & ns & ns & ns & ns \\
\hline
\end{tabular}

\section{DISCUSIÓN}

A partir de los resultados obtenidos en nuestro estudio hemos comprobado que:

a) La HC, o aumento de la PTM hasta valores que exceden los de seguridad del dializador, es más frecuente en la fase 1 que en la fase 2, como relatan en sus estudios algunos autores ${ }^{(3,4,10)}$. Además, existe mayor probabilidad de que suceda en la tercera y cuarta hora, tanto por sesión como por paciente.

b) Encontramos que hay un mayor número de sesiones que finalizan en técnica HD convencional en la fase 1 que en la fase 2. Por el contrario, en la fase 2 se produce una disminución en la frecuencia de coagulaciones del sistema.

c) Observamos que en la fase 2 se produce un aumento del líquido ultrapuro de infusión sin haber aumentado el (Qi) y una reducción significativa en la PTM. Por lo que, los niveles séricos de $\beta_{2}$ microglobulina son significativamente menores en esta fase $e^{(4)}$. Así, nos podemos plantear la hipótesis, de que mejoraríamos la eficacia de la técnica para la eliminación de medianas y grandes moléculas, si aumentáramos el Qi al pasar de técnica POS a Mixta, ya que en los procedimientos de PRE no tenemos limitación de tasa de intercambio (Qi), como ocurre en las POS, (Qi)=1/3 del flujo de san$\operatorname{gre}^{(10)}$.

d) En cuanto a la eficacia de la técnica para eliminación de pequeñas moléculas, es similar en las dos fases estudiadas, ya que no observamos variación 
en el Kt/V ni en ninguno de los otros parámetros analíticos estudiados.

e) La frecuencia de hipotensiones es similar en ambas fases, hecho no significativo.

f) Con la disminución de HC en la HDF on line mixta, así como de coagulaciones del sistema, creemos que se consigue una mejora en la seguridad de la sesión, además de una disminución de la carga de trabajo de enfermería.

\section{CONCLUSIÓN}

Cuando se produce un aumento de PTM en la técnica de HDF on line Posdilucional, la técnica Mixta se plantea como el método más eficaz ante la opción de disminuir el (Qi) en método posdilucional, ya que podemos afirmar que la frecuencia de casos en que se produce la $\mathrm{HC}$, impidiendo realizar la técnica pautada, es mayor en el método posdilucional que en el método mixto. Además, la eliminación de $\beta_{2}$ microglobulina es mayor en el método mixto que en el posdilucional y no disminuye la eficacia de la técnica.

\section{BIBLIOGRAFÍA}

1. Beerenhout $\mathrm{CH}$ et al. Optimizing renal replacement therapy-a case for online filtration therapies. Nephrol Dial Transplant 2002; 17: 2065 -2070.

2. Ledebo I. On line Hemodiafiltration: Technique and Therapy. Advances in Renal Replacement Therapies 1999; 6 (2): 195-208.
3. Canaud B, Bosc JY et al. On line hemodiafiltration. Safety and Efficacy in long-term Clinical Practice. Nephrol Dialysis Transplant 2000;15 (Supl 1).

4. Ahenholz P, Winkler RE, Ramlow W, Tiess M, Müller $\mathrm{W}$. On line hemodiafiltration with pre and post dilution: a comparison of efficacy. Int $\mathrm{J}$ Artif Organs 1997; 20: 81-90.

5. Altieri P, Sorba GB, Bolasco PG, Bostron M, Asprono E, Ferrara R, et al. On line predilution hemofiltration versus Ultrapure high-flux haemodialysis. Blod Purif 1997; 15:169-181.

6. Stiller S, Mann H. A model of solute transport through the dialyzer membrane in Hemodiafiltration. Seminars in dialysis February 1999; Vol 12 (1): 5-76.

7. Pedrini LA, Cristofaro V. On- line mixed hemodiafiltration with a Feedback for ultrafiltration control: Effect on middle-molecule removal. Kidney International 2003; vol 64 (4):1505.

8. Estrada MD. Valoración de la eficacia y seguridad de la hemodiafiltración en línea en comparación con la hemodiálisis en el tratamiento de la insuficiencia renal crónica en adultos. Agencia d'Avaluaciò de tecnologia i Recerca Mediques. (citado 18 abril 2005) URL disponible en: http:/www.aatrm.net/pdf/ct402es.pdf

9. Duranti E. Long-Term Data of Patients Changing from Standard Hemodiafiltration to Online Hemodiafiltration. Dialysis Transplant 2003; 32(2):73.

10. López Gómez JM, Lorenzo I, Jofré R. Hemodialfiltración en línea. En: Valderrabano F. Tratado de Hemodiálisis. Barcelona: Medica Jins; 1999. 